\title{
Analysis on Activities of Daily Living Ability and Its Influencing Factors of the Elderly in Community
}

\author{
Yifan Cao, Lingshan Wan, Hansheng Ding* \\ Shanghai Health Development Research Center (Shanghai Medical Information Center), Shanghai, China

\section{Email address} \\ lo129ve@qq.com (Yifan Cao), wanlingshan@shdrc.org (Lingshan Wan), dinghansheng@hotmail.com (Hansheng Ding) \\ ${ }^{*}$ Corresponding author
}

\section{To cite this article:}

Yifan Cao, Lingshan Wan, Hansheng Ding. Analysis on Activities of Daily Living Ability and Its Influencing Factors of the Elderly in Community. Science Discovery. Vol. 9, No. 3, 2021, pp. 97-100. doi: 10.11648/j.sd.20210903.12

Received: March 12, 2021; Accepted: May 10, 2021; Published: May 24, 2021

\begin{abstract}
Objective: To understand the activities of daily living (ADL) status of the elderly in Shanghai community and its influencing factors. Methods: Cluster sampling method was used to sample 8143 elderly aged 60 or above, living in a community of Jing'an District, analyze their ADL status and its influencing factors. Results: Among the individuals, about $34 \%$ of the elderly were suffered from ADL impaired, including 12\% were moderately or severely dependent. Age, education level, spouse, support from family and children, indoor facilities and self-rated health were the factors that affected ADL. Conclusion: Nearly $1 / 3$ of the elderly in the community have certain degree of ADL disability, and the risk of disability is significantly increased in the elderly aged 80 and over. Elderly with emotional support from family or children, good self-rated health or living with their spouse are tend to have a better activities of daily living. It is suggested that the community and family should pay more attention to the elderly people with risk factors, and provide them with more psychological and spiritual support and help. It can provide reference for improving the quality of life of the elderly and making relevant pension service policies.
\end{abstract}

Keywords: Community, Activities of Daily Living, Influencing Factors, Shanghai, Family Support

\section{上海市社区老年人日常生活能力状况及其影响因素分析}

曹宜璠, 万铃珊, 丁汉升

上海市卫生和健康发展研究中心（上海市医学科学技术情报研究所）, 上海, 中国

邮箱

lo129ve@qq.com（曹宜璠）, wanlingshan@shdrc.org(万铃珊）, dinghansheng@shdrc.org（丁汉升）

\begin{abstract}
摘要：目的：了解上海市某社区老年人日常生活能力（ADL）状况及其影响因素。方法: 按整群抽样法抽取上海市静 安区某街道60 岁及以上的居家老年人 8143 名, 分析调查对象的日常生活能力及其影响因素。结果: 调查对象中, 约 $34 \%$ 的老年人存在不同程度的ADL损伤，其中中度、重度依赖者占到约 $12 \%$ 。年龄、文化程度、有无配偶、家庭和子女的 支持情况、室内有无扶手和自评健康是ADL的影响因素。结论与建议: 社区中有将近 $1 / 3$ 的老年人存在不同程度的失能 情况， 80 岁及以上的高龄老人失能风险显著提高，与配偶居住、有情感支持、自评健康较好的老年人日常生活能力更 好。提示社区与家庭应更关注高龄的，有上述危险因素的老年人群，有针对性地为其提供更多心理与精神层面的支持 与帮助。为提升老年人生活质量, 制定相关养老服务政策提供参考。
\end{abstract}

关键词：社区，日常生活能力，影响因素，上海，家庭支持 


\section{1. 引言}

21世纪开始，中国老龄化快速发展，至2012年老年人 口数量已增长至 1.94 亿, 预计至 2022 年人口老龄化水平将 升至 $18.5 \% ， 2050$ 年, 老龄化水平将达到 $34.1 \%[1]$ 。

上海作为中国经济发达的特大型城市, 人口老龄化的 现象更为突出。统计数据显示, 2019年上海户籍人口期望 寿命83.66岁, 其中男性81.27岁, 女性86.14岁[2]。截至2019 年底, 上海市 60 岁及以上老年人口 518.12 万人, 占总人口 的 $35.2 \%$ 。

随着预期寿命的提高, 丧失日常生活能力的老人越来 越多, 而老年人日常生活能力是反映老年人健康状况的客 观指标之一,也在一定程度上体现了老年人的健康生命质 量水平 [3-4]。了解社区老年人日常生活能力状况及其影响 因素, 有助于维护增进老年人生活质量, 为制定相关的养 老服务政策提供参考依据。

\section{2. 研究方法}

\section{1. 调查对象}

本研究采用整群抽样的方式对上海市静安区某街道 60 岁及以上老年人进行调查, 最终回收有效问卷 8143 份。

\section{2. 调查工具}

采用的调查表为上海市卫生和健康发展研究中心编 制的《上海市老年照护统一需求评估调查表》。内容包括 人口学特征、日常生活能力量表 (Activity of Daily Living
Scale, ADL ),具体分值采用巴氏日常生活能力指数 [5] (Barthel Index of Activities of Daily Living) 表示, 包括进 食、洗澡、修饰（洗脸、刷牙、刮脸、梳头）、穿脱衣物、 大便、小便、用则、床椅转移、平地移动、上下楼梯等 10 个方面。本研究采用日常生活能力 (Activity of Daily Living，以下简称“ADL”）来评估上海市老年人的老年照 护的需求, 每个项目根据是否需要帮助及其帮助程度分 为 $0 、 5 、 10 、 15$ 分 4 个功能等级, 总分为 100 分, 得分越高 其自理能力越强, 依赖性越小。得分 100 分无需依赖, 得 分60分以上者为轻度依赖, 41-60分者中度依赖, 得分 40 分及以下者为重度依赖。

\section{3. 统计学方法}

采用 SAS9.4 软件进行统计学分析、Cumulative Logistic回归分析影响因素, 并计算不同暴露因素下的OR 值。

\section{3. 结果}

\section{1. 研究对象日常生活能力情况}

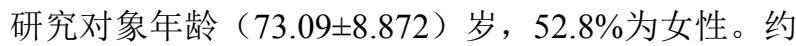
$34 \%$ 的老年人存在不同程度的ADL损伤, 其中中度、重度 依赖者占到约 $12 \%$ 。随着年龄增加, ADL轻度级以上的依 赖比例也随之增加。女性ADL受损比例要高于男性。失能 者 (中度及以上依赖) 中文化程度较低的依赖比例高于文 化程度高的, 具体占比见表 1 。

表1 社区老年人基本情况及ADL依赖程度（\%）。

\begin{tabular}{|c|c|c|c|c|c|c|}
\hline 项目 & & 人数占比 & 无需依赖 & 轻度依赖 & 中度依赖 & 重度依赖 \\
\hline 总数 $(n=8143)$ & & 100.0 & 65.75 & 22.72 & 6.85 & 4.68 \\
\hline \multirow{3}{*}{ 年龄（岁）(n=8143) } & $60 \sim$ & 44.8 & 85.02 & 12.53 & 1.59 & 0.85 \\
\hline & $70 \sim$ & 26.9 & 70.22 & 20.06 & 6.38 & 3.33 \\
\hline & $80 \sim$ & 28.3 & 30.99 & 41.36 & 15.63 & 12.02 \\
\hline \multirow{2}{*}{ 性别（n=8124） } & 男 & 47.2 & 67.84 & 21.49 & 6.34 & 4.33 \\
\hline & 女 & 52.8 & 63.80 & 23.89 & 7.32 & 4.99 \\
\hline \multirow{5}{*}{ 文化程度（n=8087） } & 文盲 & 3.7 & 33.33 & 28.96 & 14.48 & 23.23 \\
\hline & 小学 & 22.4 & 46.39 & 34.91 & 11.31 & 7.39 \\
\hline & 初中 & 36.3 & 74.11 & 14.38 & 6.44 & 5.08 \\
\hline & 高中 & 30.5 & 74.02 & 21.60 & 3.69 & 0.69 \\
\hline & 大学及以上 & 7.2 & 68.22 & 26.42 & 4.66 & 0.69 \\
\hline \multirow{4}{*}{ 主要经济来源 (n=8031) } & 退休金 & 99.5 & 65.91 & 22.61 & 6.91 & 4.57 \\
\hline & 子女补贴 & 0.3 & 41.67 & 41.67 & 8.33 & 8.33 \\
\hline & 亲友赞助 & 0.0 & 100 & 0.00 & 0.00 & 0.00 \\
\hline & 其他补贴 & 0.2 & 83.33 & 5.56 & 0.00 & 11.11 \\
\hline \multirow[t]{2}{*}{ 有无配偶（n=8001） } & 有 & 80.1 & 71.77 & 19.51 & 5.49 & 3.23 \\
\hline & 无 & 19.9 & 41.66 & 35.76 & 12.36 & 10.23 \\
\hline \multirow{4}{*}{$\begin{array}{l}\text { 家庭和子女的支持情况如 } \\
\text { 何 }(n=8106)\end{array}$} & 提供物质和情感支持 & 88.9 & 63.53 & 24.04 & 7.5 & 4.93 \\
\hline & 仅提供物质支持 & 1.8 & 59.46 & 21.62 & 8.11 & 10.81 \\
\hline & 仅提供情感支持 & 8.7 & 89.32 & 10.26 & 0.00 & 0.00 \\
\hline & 没有家庭或其他情况 & 0.6 & 80.77 & 9.62 & 5.77 & 3.85 \\
\hline
\end{tabular}




\begin{tabular}{|c|c|c|c|c|c|c|}
\hline 项目 & & 人数占比 & 无需依赖 & 轻度依赖 & 中度依赖 & 重度依赖 \\
\hline 自评健康（ $\mathrm{n}=8122 ）$ & 好 & 92.3 & 68.76 & 23.47 & 6.16 & 1.61 \\
\hline & 差 & 7.7 & 30.51 & 13.42 & 15.18 & 40.89 \\
\hline
\end{tabular}

\section{2. 老年人日常生活能力影响因素}

结果显示, 老年人的日常生活能力与其年龄、文化程度、有无配偶、家庭和子女的支持情况、室内环境情况以及 自评健康因素有关。（表2）

表2 老年人日常生活能力的影响因素。

\begin{tabular}{|c|c|c|c|c|c|c|}
\hline \multirow{2}{*}{ 项目 } & & \multicolumn{5}{|c|}{ Logistic回归 } \\
\hline & & b & $\mathbf{P}$ & $\chi^{2}$ & OR & $95 \% \mathrm{Cl}$ \\
\hline \multirow{3}{*}{ 年龄/岁 (参照60 69) } & $70 \sim 79$ & 0.921 & $<.0001$ & 198.873 & 2.511 & $2.209 \sim 2.853$ \\
\hline & $80 \sim$ & 2.512 & $<.0001$ & 1623.720 & 12.325 & $10.907 \sim 13.926$ \\
\hline & 小学 & -0.813 & $<.0001$ & 50.826 & 0.444 & $0.355 \sim 0.555$ \\
\hline \multirow{3}{*}{ 文化程度（参照文盲） } & 初中 & -1.907 & $<.0001$ & 281.069 & 0.149 & $0.119 \sim 0.186$ \\
\hline & 高中 & -2.004 & $<.0001$ & 300.336 & 0.135 & $0.107 \sim 0.169$ \\
\hline & 大学及以上 & -1.743 & $<.0001$ & 158.496 & 0.175 & $0.133 \sim 0.229$ \\
\hline 有无配偶（参照有配偶） & 无 & 1.232 & $<.0001$ & 512.506 & 3.429 & $3.082 \sim 3.815$ \\
\hline \multirow{3}{*}{$\begin{array}{l}\text { 家庭和子女的支持情况如何（参照提供物质 } \\
\text { 和情感支持） }\end{array}$} & 仅提供物质支持 & 0.265 & 0.1011 & 2.688 & 1.304 & $0.949 \sim 1.790$ \\
\hline & 仅提供情感支持 & -1.596 & $<.0001$ & 161.239 & 0.203 & $0.159 \sim 0.259$ \\
\hline & 没有家庭或其他情况 & -0.829 & 0.0164 & 5.757 & 0.437 & $0.222 \sim 0.859$ \\
\hline 室内有无扶手（参照有扶手） & 无 & -1.252 & $<.0001$ & 232.789 & 0.286 & $0.244 \sim 0.336$ \\
\hline 自评健康（参照自评健康好） & 差 & 2.563 & $<.0001$ & 996.976 & 12.976 & $11.067 \sim 15.213$ \\
\hline
\end{tabular}

\section{4. 讨论}

研究显示，社区中有约 $12 \%$ 的老年人为中度及以上 的依赖者, 且随着年龄增加, 老年人ADL逐渐下降, 需 要照护的比例也随之增加, 其影响在 80 岁及以上老年人 尤为显著。上海老龄化人口多, 高龄老人占比高, 2019 年底, 80 岁及以上高龄老年人口 81.98 万人, 占 60 岁及以 上老年人口的 $15.8 \%$, 占总人口的 $5.6 \%[2]$ 。老年人群因 $\mathrm{ADL}$ 受限而导致的长期照护需求会越来越凸显 $[6-8]$ 。与 既往的部分研究相似 [9-10], 文化程度较高的老年人, 日 常生活能力也相对较好 [11]。可能是因为文化程度较高的 老年人阅读及理解能力较好, 具有较强的自我保健和疾 病预防意识。

研究还发现, 无配偶的老年人其日常生活能力受损的 风险约为有配偶老年人的 3 倍, 这可能是由于在日常生活 照料中, 配偶的支持对老人能起到重要的作用 [12-13], 通 过配偶的监督以及支持, 有利于影响老人建立良好健康的 生活方式[14]。仅提供情感支持家庭中的老年人, 日常生 活能力更好, 也提示了情感支持对老年人的重要性。

从外部因素来看, 家中无扶手的老年人日常生活能力 更好, 可能是本身这部分老人身体状况就较好, 从而无需 安装扶手。从老年人自身健康评价来看, 自我评价差的老 年人, 日常生活能力也相对较差。一方面可能是本身老年 人实际的躯体健康状况较差, 另一方面也有可能是由于老 年人心理及情感上的负面影响, 心理健康水平的弱化而表 现出的消极情绪, 而不仅仅是因为躯体功能状况差的原因 [15]，提示老年人心理健康状况也值得关注。

\section{5. 结论与建议}

随着年龄增加, 老年人失能风险随之增加。在 80 岁以上 的高龄老人中尤为显著。本研究还发现与配偶居住的老年人 失能风险较低、有情感支持的老年人日常生活能力更好。因 此, 建议社区加强对 80 岁及以上高龄及无配偶老年人的关怀 与关注, 将对改善老年人的自理能力起到至关重要的作用。

除此以外, 笔者呼吁应在现有的服务体系中考虑老年 人心理健康的维持, 鼓励家庭给予老人更多情感方面的支 持与慰藉。将有助于提高老年人生活质量, 延缓老年人日 常生活能力的下降。

\section{致谢}

本文为上海市第四轮公共卫生体系建设三年行动计 划项目（上海市医养结合体系建设研究, GWIV-37）。

\section{参考文献}

[1] 总报告起草组, 李志宏. 国家应对人口老龄化战略研究总 报告[J].老龄科学研究, 2015(03):6-40.

[2] 上海市老龄科学研究中心, 2019 年上海市老年人口和老龄 事 业 监 测 统计信息 http://wsjkw.sh.gov.cn/tjsj2/20200527/06873e6ec8f54a158c2 5475dbbb574a6.html [EB/OL].[2021-03-06]. 
[3] 董毅,郭继志,胡善菊.山东省城乡社区老人生命质量状况及 影响因素探讨 $[J]$.医学与社会,2015,28(11):58-60.

[4] 肖慧欣,林诗竹,林祺,等.不同养老形式的老年人日常生活能 力比较[J].中国老年学杂志,2017,37(04):999-1000.

[5] Collin C, Wade DT, Davies S, Horne V. The Bathel ADL index: a reliability study.IntDisabilStud.1988,10(2):61-63)

[6] 纪经海. 老年人日常活动能力影响因素调查及研究 [J]. 影 像研究与医学应用, 2017, 001(011):250-251.

[7] 刘沝,王建华,杨姗姗, 等.北京市社区老年人群日常活动能力 状况及城乡比较 [J].中华流行病学杂志,2018,39(3):268-272.

[8] 陈晶, 段曹辉. 中国老年人日常活动能力的影响因素 基于 CHARLS 数据的分析 [J]. 公共卫生与预防医学, 2017,28(3):68-71.

[9] 徐小凤,陈茜,赵艺璞,等.我国西部地区居家老年人生活自理 能力调查分析 $[\mathrm{J}]$. 北京大学学报 (医学 版),2018,50(03):457-462.
[10] 李聪,张小丽,张盼,等.蛙埠市老年人日常生活能力现状及影 响因素[J].中国公共卫生,2017,33(06):991-993.

[11] 翟晓婷,黄丽妹,阮晔,黄哲宙,费吉辉,丁国梅,齐士格,王志会. 上海市松江区老年人日常生活能力现状 $[\mathrm{J}]$. 上海预防医 学,2018,30(04):320-323+327.

[12] 高利平, 袁长海, 刘保锋, 等. 山东省老年人生活自理能 力及影响因素分析 $(\mathrm{J})$. 中国公共卫生，2010；26( 11)： 1359-61.

[13] 仲亚琴, 王健, 张国杰. 老年人日常生活能力现状及影响 因素分析 ( $\mathrm{J} 〕$. 现代预防医学, 2014; 41( 5): 852-5.

[14] 刘建春,郝晓宁,薄涛,等.空巢老人生活自理能力现状及其社 会支持体系分析[J].中国卫生经济,2014,33(07):68-71.

[15] 刘恒,巢健茜,杨迎春,等.老年人自评健康影响因素分析及程 度比较[J]. 中国全科医学, 2009, 12(13). 